Z. klin. Chem. u. klin. Biochem.

8. Jg., S. 269-272, Mai 1970

\title{
Eine Vorrichtung zur Konstanthaltung kleiner Substratkonzentrationen bei kinetischen Messungen mit dem pH-Staten
}

\author{
Von Irene RossmanN, F. Körber und P. Stegmund \\ Aus dem Physiologisch-Chemischen Institut der Freien Universität Berlin
}

(Eingegangen am 10. März 1970)

\begin{abstract}
Ein pH-Stat wird mit einer Bürettenkombination versehen, mit der dem Bestimmungsansatz eine Substratlösung mit gleicher Geschwindigkeit zugeführt wird wie das zur Neutralisation benötigte Titrationsmittel. Bei gleichen molaren Konzentrationen von Substratlösung und Titrationsmittel wird dadurch die Substratkonzentration im Bestimmungsansatz konstant gehalten. Auch bci kleinen Substratkonzentrationen werden so für einige Minuten gerade Zeit-Umsatz-Kurven erhalten. Die Methode wird bei der Bestimmung der $\mathrm{K}_{\mathrm{m}}$-Werte von Penicillinase und Lactatdehydrogenase erprobt.
\end{abstract}

\section{An apparatus for maintaining the constancy of low substrate concentrations in kinetic measurements with a $p H$-stat}

A pH-stat is equipped with a combination of burettes, arranged so that substrate solution and the titration reagent required for neutralisation, are added to the reaction mixture at the same rate. If the molar concentrations of the substrate solution and the titration reagent are the same, the substrate concentration in the reaction mixture remains constant. In this way, even for low substrate concentrations, rectilinear reaction-time curves are obtained over a few minutes. The method has been tested in the determination of the $\mathrm{K}_{\mathrm{m}}$-values of penicillinase and lactate dehydrogenase.

Reaktionen, bei denen Wasserstoffionen verbraucht oder freigesetzt werden, können mit der pH-Stat-Methode verfolgt werden (1). Bei dieser Methode wird die Zufuhr des zur Konstanthaltung eines vorgegebenen $\mathrm{pH}$-Wertes benötigten Titrationsmittels aus einer mechanisch betriebenen Bürette durch das Potential der Glaselektrode gesteuert. Ein mechanischer Schreiber registriert den zeitlichen Verlauf dieser Zugabe. Die Methode wird z. B. bei der Messung von Enzymaktivitäten von Esterasen und Amidasen benutzt. Sollen mit solchen Messungen Michaeliskonstanten ermittelt werden, so müssen oft Reaktionsgeschwindigkeiten bei sehr kleinen Substratkonzentrationen gemessen werden. $\mathrm{Da}$ andererseits bei genaueren Messungen die Konzentration des Titrationsmittels nicht kleiner als $10 \mathrm{mN}$ und der Verbrauch pro Minute nicht kleiner als $20 \mu l$ sein sollte, muß die Enzymkonzentration entsprechend gewählt werden. Bei solchen Messungen nimmt dann die Substratkonzentration im Ansatz und dadurch die Reaktionsgeschwindigkeit sehr schnell $a b$, und es werden stark gekrümmte Zeit-Umsatz-Kurven erhalten, deren Auswertung mit erheblichen Fehlern verbunden ist.

Der Versuch, dieser Abnabme der Substratkonzentration im Ansatz dadurch zu begegnen, daß dem Titrationsmittel die gleiche molare Menge Substrat zugesetzt wird, scheitert meist daran, daß die üblichen Substrate im Titrationsmittel nicht beständig sind.

Wir haben deshalb eine Vorrichtung gebaut, mit der Titrationsmittel und Substratlösung gleicher Konzentrationen simultan aus zwei Kolbenbüretten mit gleicher Geschwindigkeit zugeführt werden.

\section{Material und Geräte}

Extinktionsmessungen erfolgten mit dem Photometer Eppendorf (Cd-Lampe, Filter Cd $480 \mathrm{~nm}$ ) oder mit dem Spektralphotometer PMQ II (Zeiss). Die pH-Stat-Methode wurde mit der Titrationseinrichtung von Radiometer Kopenhagen, bestehend aus Büretteneinheit SBU 1 a, Rührmotor SMP 1a, Titrator TTT 1c und Titrigraph SBR 2c durchgeführt. Die zusätzliche Anordnung ist im Text beschrieben (Abb. 1).

Das verwendete Benzylpenicillin (Natriumsalz) war Testpenicillin (Hoechst, $1650 \mathrm{E} / \mathrm{mg}$ ). Als Penicillinase ${ }^{1}$ ) wurde das Handelspräparat Neutrapen (Riker, Northridge, California), das als Lyophilisat in Ampullen erhältlich ist, verwendet.

Lactatdehydrogenase ${ }^{1}$ )-Suspension (aus Kaninchenmuskel, Bande 5 , nativ, $5 \mathrm{mg} / \mathrm{m} /$ ) und NADH waren Präparate ron Boehringer, Mannheim.

Die übrigen Chemikalien waren analysenreine Substanzen des Handels, zur Bereitung der Lösungen wurde bidest. Wasser aus Glasapparaturen benutzt.

Penicillinaselösung: Der Inhalt einer Ampulle Neutrapen wurde in $2 \mathrm{~m} /$ bidest. Wasser gelöst. Die so erhaltene Lösung enthielt etwa $670 \mathrm{U} / \mathrm{m} l^{2}$ ). Vor der Verwendung wurde die Lösung 1:100 mit $1 \mathrm{~mm}$ Phosphatpuffer, $\mathrm{pH}$ 6,8, der $0,5 \%$ Gelatine enthielt, verdünnt. Diese Lösung ist im Eisbad für einige Stunden mit unveränderter Aktivität haltbar.

Herstellung dẹ $20 \mathrm{~mm}$ Penicillinlösıng für die Beschickung der Kolbenbürette: Eine Kolbenbürette der Büretteneinheit (Abb. 1) wurde mit einer Pcnicillinlösung, die etwas konzentrierter als $20 \mathrm{~mm}$ war (200 mg Penicillin in $20 \mathrm{ml} 1 \mathrm{~mm}$ Phosphatpuffer $\mathrm{pH}$

1) Enzyme: Penicillinase $=$ Pcnicillin Amido- $\beta$-lactambydrolase (EC 3.5.2.6)

Lactatdehydrogenase $=$ L-I_actat: NAD Oxydoreduktase (EC 1.1.1.27)

2) Angabe in internationalen Enzymcinheiten: Substratumsatz in $\mu \mathrm{Mol} / \mathrm{Min}$. bei $25^{\circ}$. Davon zu unterscheiden sind die (hier nicht benutzten) Penicillinase-Einheiten nach G. B. Lr:vy (Nature London 166, 740 [1950]): Eine Einheit ist hier dic Enzymmenge, die eine Einheit Penicillin in einer Minutc bei $25^{\circ}$ und $\mathrm{pH} 7,0$ spaltet. 

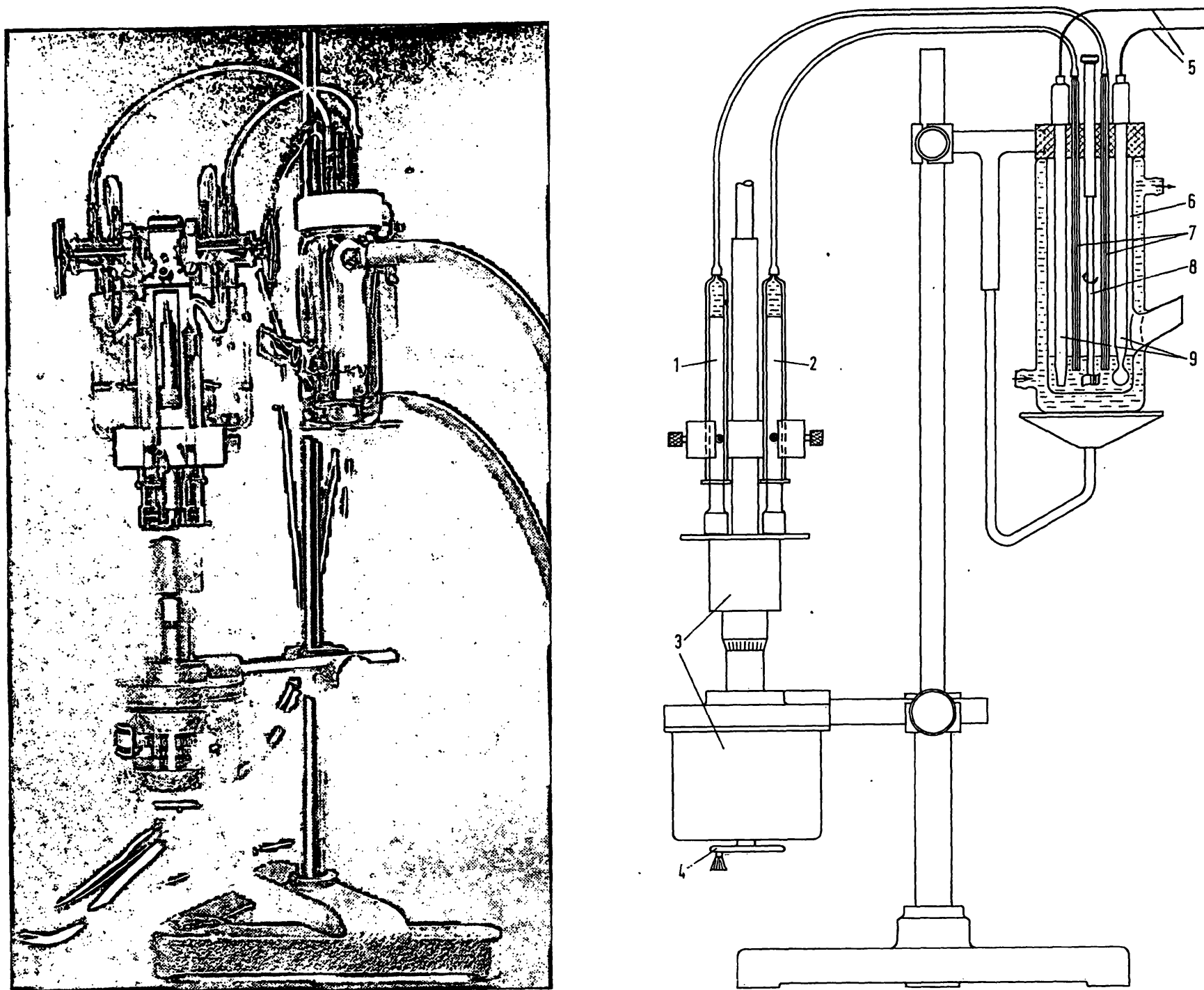

Abb. 1

Büretteneinheit für den simultanen Betrieb eines pH-Staten mit zwei 1-ml-Kolbenbüretten $1=$ Kolbenbürette für Titrationsmittel
$2=$ Kolbenbürette für Substratlösung
$3=$ mechanischer Bürettenvorschub, mit Schreiber
$4=$ gekoppelt $5=$ Anschluß zum pH-Staten $6=$ temperiertes Reaktionsgefäß $7=$ Einleitungskapillaren $8=$ Rührer $9=$ Elektroden

$6,8)$ beschickt. $0,5 \mathrm{~m} /$ dieser Iösung wurden (durch Betätigung des Handrades) zu 10,0 ml $1 \mathrm{~mm}$ Phosphatpuffer $\mathrm{pH}$ 6,8 gegeben. Durch Zusatz von $20 \mu l$ unverdünnter Penicillinaselösung wurde das Penicillin zu Benzylpenicillosäure hydrolysiert und diese mit $20 \mathrm{mN} \mathrm{NaOH}$ aus der zweiten Kolbenbürette des $\mathrm{pH}$-Staten wieder auf pH 6,8 titriert. Die Konzentration der Penicillinlösung wurde dann durch Verdünnen dem Titex der $20 \mathrm{mN} \mathrm{NaOH}$ angeglichen.

Lactatdebydrogenaselösung: $100 \mathrm{mg}$ Gelatine wurden in $20 \mathrm{~m} l 1 \mathrm{~mm}$ Trispuffer $\mathrm{pH} 7,5$ unter Erwärmen gelöst und nach Abkühlen und $\mathrm{pH}-$ Kontrolle $40 \mu l$ Lactatdehydrogenase $(5 \mathrm{mg} / \mathrm{m} l)$ zugefügt. Die Lösung ist im Eisbad einige Stunden mit unveränderter Aktivität haltbar.

Herstellung der $10 \mathrm{~mm} N A D H / 10 \mathrm{~mm}$ Natriumpyruvatlösung für die Beschickung der Kolbenbürette: $22 \mathrm{mg}$ Natriumpyruvat wurden im 20-ml-Meßkolben in 10,0 m/ $20 \mathrm{~mm}$ NADH-Lösung (s.u.) gelöst und nach Einstellen des pH-Wertes auf $\mathrm{pH} 7,5$ mit bidest. Wasser auf $20,0 \mathrm{~m} l$ aufgefüllt.

$20 \mathrm{~mm} N A D H$-Lösung: Eine Probe einer NADH-Lösung, die etwas konzentrierter als $20 \mathrm{~mm}$ war $(256 \mathrm{mg} \mathrm{NADH}$ in $15 \mathrm{~m} l \mathrm{mit}$ einem Tropfen verd. $\mathrm{NaOH}$ alkalisiertem bidest. Wasser) wurde mit $1 \mathrm{~mm}$ Trispuffer $\mathrm{pH}$ 7,5 1:20 verdünnt (etwa $1 \mathrm{~mm}$ NADH). Die Konzentration dieser Lösung wurde photometrisch (d =
$0,5 \mathrm{~cm}, \lambda=366 \mathrm{~nm}$ ) bestimmt und die Konzentration der StammLösung entsprechend dem Meßergebnis durch Verdünnen korrigiert.

\section{Beschreibung der Methode und Diskussion}

Zunächst wurde überprüft, mit welcher Genauigkeit die Volumina der Lösungen, die aus den Kolbenbüretten der Büretteneinheit beim simultanen Hub der Kolben abgegeben werden, übereinstimmen. Hierzu wurden beide Kolbenbüretten mit der gleichen $0,2 \mathrm{mM} \mathrm{NH}_{4} \mathrm{Fe}\left(\mathrm{SO}_{4}\right)_{2}-$ Lösung gefüllt. In zwei 4-cm-Küvetten wurden zu je $10 \mathrm{ml} 0,1 \mathrm{M}$ KSCN-Lösung über Kapillarschläuche in Schritten von 50 Skalenteilen (Nonius der Büretteneinheit) simultan aus beiden Bürettenspritzen die $\mathrm{Fe}$ (III)Lösungen in je eine Küvette gepumpt. Nach dem Mischen wurden die Extinktionen bei $\lambda=480 \mathrm{~nm}$ (entspricht dem Absorptionsmaximum des Eisen[III]Thiocyanat-Komplexes) am Eppendorf-Photometer (Cd-Lampe) gegen Wasser gemessen. Die gemessenen 


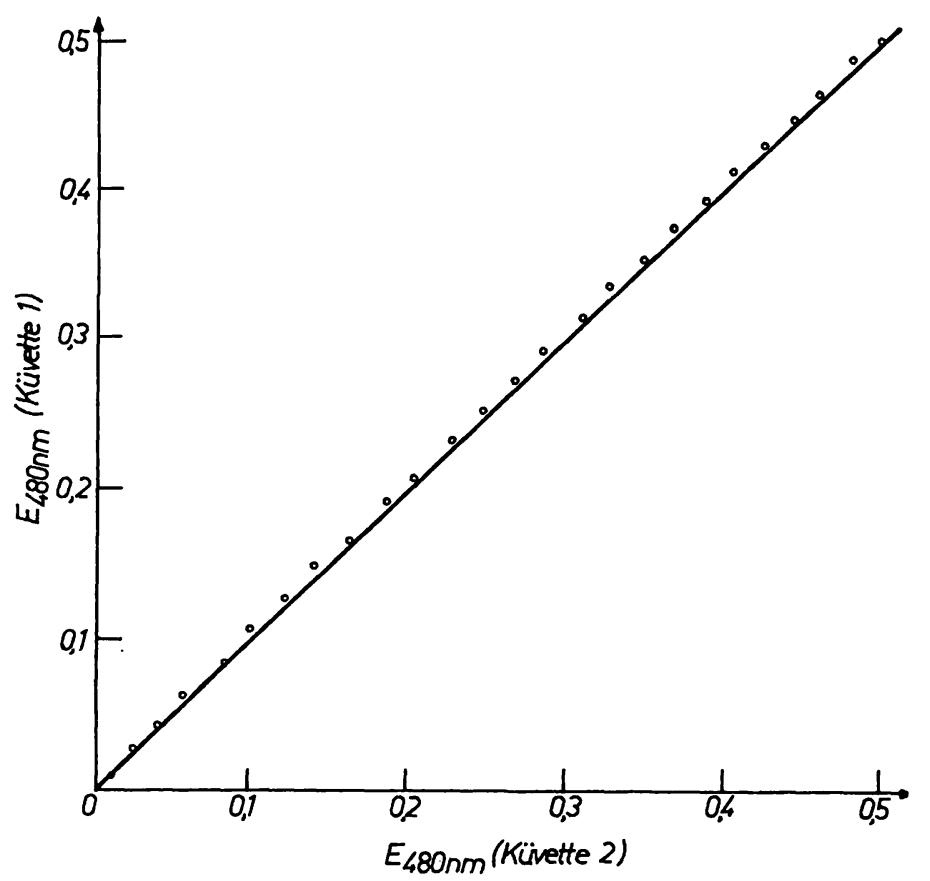

Abb. 2

Prüfung der Übereinstimmung der beiden Kolbenbüretten: aus beiden Büretten wurde die gleiche Eisen(III)-Lösung in je eine mit NaSCNLösung beschickte Küvette gegeben und die Bildung des Eisen-Rhodanidkomplexes photometrisch verfolgt. Die für die beiden Küvetten erhaltenen Extinktionswerte sind gegeneinander aufgetragen. Die eingezeichnete Gerade entspricht der genauen simultanen Förderung gleicher Teilvolumina

Extinktionen wurden gegeneinander aufgetragen. Das Diagramm (Abb. 2) zeigt, daß die Meßpunkte einer Geraden mit der Steigung $\operatorname{tg} \alpha=1$ folgen, d.h., da $\beta$ die Kolbenbüretten im gesamten Bereich mit ausreichender Genauigkeit übereinstimmen.

Penicillinase spaltet im Penicillin die Amidbindung des Lactamringes. $\mathrm{Da}$ die entstehende NH-Gruppe im Thiazolidinring steht, besitzt sie nur geringe Basizität und die Titration der durch Dissoziation der Carboxylgruppe entstehenden Wasserstoffionen ist ein $\mathrm{MaB}$ für den Fortschritt der Reaktion. Das wurde von CITRI und $\mathrm{ZYK}_{\mathrm{Y}}$ (2) zur Bestimmung der Penicillinase ${ }^{3}$ ) mit der $\mathrm{pH}$-Stat-Methode benutzt.

Einige Penicillinase-Spezies besitzen sehr kleine Michaeliskonstanten, so daß nur die hier beschriebene Modifi-

3) CITRI und $\mathrm{ZYK}$ exhielten jedoch zu niedrige Werte, da sie auf pH 6,0 titrierten - einen pH-Wert, bei dem der Thiazolidin-N noch teilweise protoniert ist. kation der Methode für die Bestimmung dieser Konstanten geeignet ist.

Zur Bestimmung der Penicillinaseaktivität wurde das auf $25^{\circ}$ temperierte doppelwandige Reaktionsgefäß mit $2,0 \mathrm{~m} l 1 \mathrm{~mm}$ Phosphatpuffer $\mathrm{pH}$ 6,8, $1,0 \mathrm{~m} / 0,5$ proz. Gelatinelösung im gleichen Phosphatpuffer und $200 \mu \mathrm{l}$ der verdünnten Penicillinaselösung beschickt. Der $\mathrm{pH}$ Stat wurde bei stehendem Vorschub so eingestellt, daß die $\mathrm{pH}$-Anzeige des Gerätes mit dem $\mathrm{pH}$-Wert der Lösung $(6,8)$ übereinstimmte. Dann wurde durch $\mathrm{Zu}-$ gabe von $1,0 \mathrm{~m} l$ einer Penicillinlösung geeigneter Konzentration durch den seitlichen Einfüllstutzen die Reaktion gestartet; der Schreiber des Gerätes zeichnet dann den Hub der Bürettenkolben, der der zur Aufrechterhaltung des vorgegebenen $\mathrm{pH}$-Wertes notwendigen Laugenzugabe entspricht, auf.

Die Parallelschaltung einer mit der Penicillinlösung gleicher molarer Konzentration beschickten Kolbenbürette bewirkt den Ersatz des gespaltenen Substrats und damit die Aufrechterhaltung der vorgegebenen Substratkonzentration, wenn man von dem Verdünnungseffekt absieht. Das bedeutet, daß die Reaktionsgeschwindigkeit wenigstens für einige Minuten konstant ist. Der Versuch wurde mit Penicillinkonzentrationen von 200, 150, 100, 50,40 und $30 \mu \mathrm{M}$ im Reaktionsgefäß durchgeführt.

Die erhaltenen Kurven sind in Abbildung 3a wiedergegeben. Zur Auswertung wurden die geraden Anfangsteile verlängert. Nur bei der kleinsten Konzentration ist das Anlegen einer Tangente erforderlich und unsicher. Im Lineweaver-Burk-Diagramm (Abb. 4a) liegt jedoch auch dieser Wert für $30 \mu \mathrm{M}$ noch nahe an der Geraden.

Bei der Bestimmung der Aktivität und der Michaeliskonstanten von Lactatdebydrogenase ist natürlich die photometrische Messung bei 340 bzw. 366 nm (3) die Methode der Wahl. Indessen erscheint uns dieses so gut untersuchte Enzym geeignet, um die hier beschriebene Versuchsanordnung zu exproben. Das benutzte Enzympräparat war Lactatdehydrogenase aus Kaninchenmuskel, Bande 5 (nativ); als Substrat, dessen Konzentration von Ansatz zu Ansatz variierte, wurde NADH gewählt, die Pyruvatkonzentration war in allen Ansätzen $1 \mathrm{~mm}$. Als Titrationsflüssigkeit dienten einerseits $10 \mathrm{mN} \mathrm{HCl}$ (erste Kolbenbürette) und andererseits eine Lösung, die $10 \mathrm{~mm}$ an
Abb. 3

Mit dem pH-Staten erhaltene Zeit-Üm, satz-Kurven bei gesteuerter Substrata) Penicillinase

b) Lactatdehydrogenase

Ansätze und Reaktionsbedingungen siehe Methodik. Die Zahlen an den Kurven geben die Substratkonzentrationen $(\mu \mathrm{M})$ in den entsprechenden Ansätzen an
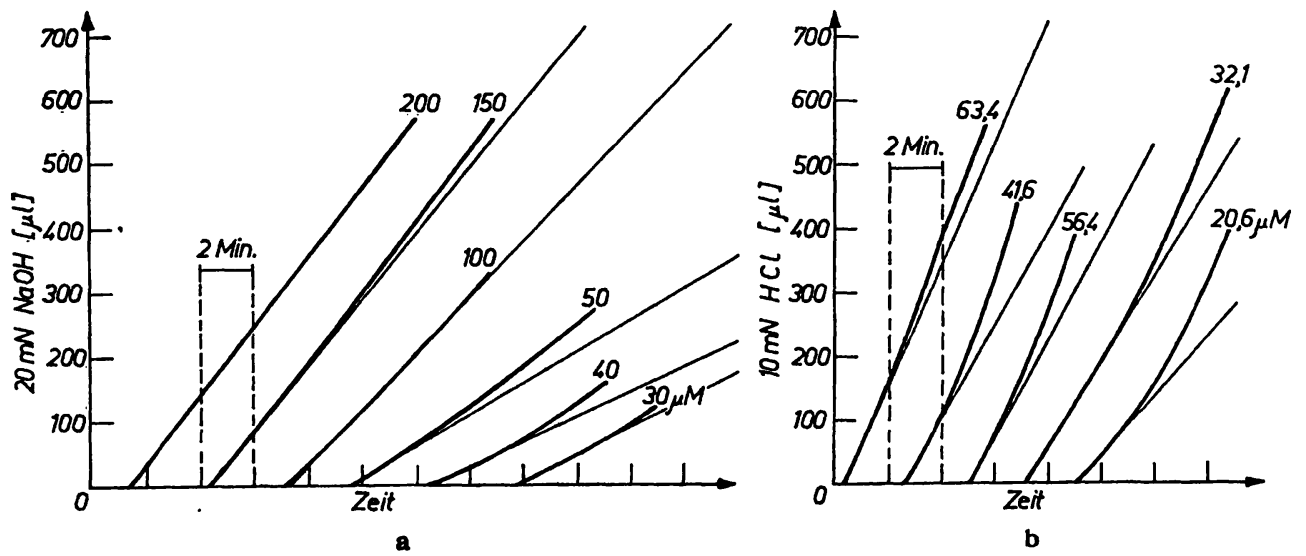


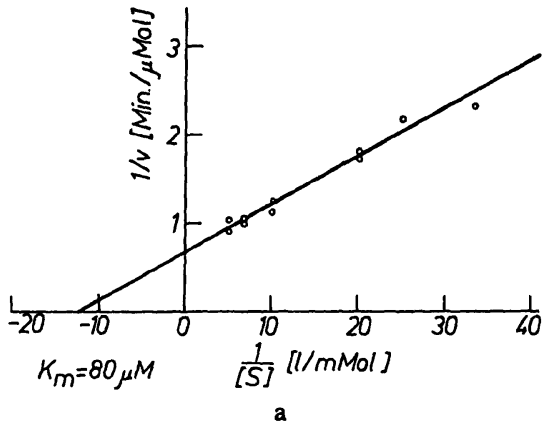

a

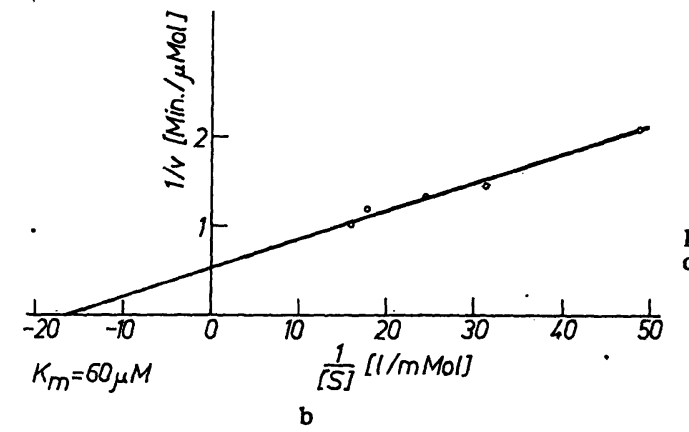

b
Abb. 4

Diagramme nach Lineweaver-Burk aus den Zeit-Umsatz-Kurven der Abbildung 3

a) für Penicillinase

b) für Lactatdehydrogenase
NADH und an Pyruvat war (zweite Kolbenbürette), wodurch erreicht wurde, daß beide Substrate im Maß ihres Verbrauchs nachgeliefert wurden.

Für den Versuch wurde das auf $25^{\circ}$ temperierte Reaktionsgefäß mit $3,0 \mathrm{~m} / 1,67 \mathrm{mM}$ Natriumpyruvat-Lösung und $1,0 \mathrm{~m} l$ Lactatdehydrogenaselösung beschickt. Bei stehendem Vorschub wurde die pH-Anzeige des Geräts auf den $\mathrm{pH}$-Wert der Lösung ( $\mathrm{pH} 7,5)$ eingestellt, und die Reaktion durch Zugabe von 1,0 ml einer NADHLösung geeigneter Konzentration durch den seitlichen Einfüllstutzen gestartet. Die Endkonzentrationen betrugen dann für Pyruvat $1 \mathrm{~mm}$, für Lactatdehydrogenase $2 \mu \mathrm{g} / \mathrm{m} l$. Die Konzentrationen der zugegebenen NADHLösung waren durch Extinktionsmessungen kontrolliert worden. Aus diesen Werten wurden die Konzentrationen im Ansatz zu 63,4,56,4,41,0,32,1 und 20,6 $\mu \mathrm{M}$ berechnet. Aus den Anfangssteigungen der am Beginn nahezu geradlinigen Zeit-Umsatz-Kurven (Abb. 3b) wurde der Substratumsatz in $\mu \mathrm{Mol} / \mathrm{Min}$. berechnet. Die Reziprokwerte wurden im Lineweaver-Burk-Diagramm gegen die zugehörigen reziproken NADH-Konzentrationen der Ansätze aufgetragen (Abb. 4b). Aus dem Diagramm kann man ersehen, daß auch bei den kleinsten $\mathrm{NADH}-$ Konzentrationen noch brauchbare Werte erhalten werden ${ }^{4}$ ).

Wie aus den Abbildungen $3 a$ und $b$ zu entnehmen ist, werden vor allem bei den kleinsten Konzentrationen nur während der ersten Minuten geradlinige ZeitUmsatz-Kurven erhalten. Bei Kurven, die unter gleichen Bedingungen mit der einfachen $\mathrm{pH}$-Stat-Methode - ohne Substratzufuhr - zu erhalten sind, wäre die Reaktion jedoch schon nach Zufuhr von etwa $10 \mu \mathrm{l}$ Titrationsmittel zum Stillstand gekommen. Für Reaktionszeiten, die mehr als einige Minuten betragen, sind aber auch mit der hier beschriebenen Methode keine Geraden zu erhalten: $\mathrm{Da}$ nämlich die Konzentrationen der Zufuhrlösungen 2-3 Zehnerpotenzen größer als die im Ansatz sind, führen bereits geringfügige Abweichungen in der Zufuhr dieser Lösung vom Substratverbrauch bei den kleineren Konzentrationen schnell zu deren Änderung und damit auch zur Änderung der Reaktionsgeschwindigkeit.

Wir danken Herrn Mechaniker L. Schreyer für den Bau der Büretteneinheit, Herm Dr. KerrelHack, Kettelhack Riker Pharma GmbH., 428 Borken (Westf.), für die Úberlassung von Penicillinase und den Farbwerken Hoechst für die Uberlassung von Testpenicillin.

4) Der hier erhaltene $K_{m}$-Wert ist nicht ohne weiteres mit in der Literatur angegebenen Werten zu vergleichen, da diese sich auf das am aktiven Zentrum mit Pyruvat gesättigte Enzymprotein beziehen.

Wegen der Hemmung durch größere Pyruvatkonzentrationen können diese Werte nur durch Extrapolation aus mehreren Meßreihen bei verschiedenen Pyruvatkonzentrationen erhalten werden (4).

\section{Literatur}

1. Jacobsen, C. F., J. LÉonis, K. Linderstrøm-LANG und M. Otresen, The pH-Stat and Its Use in Biochemistry, Meth. biochem. Analysis 4, 171 (1957). - 2. Crrrr, N. und N. Zrk, Biochim. biophysica Acta Amsterdam 99, 427 (1965). - 3. BERG-
MEYER, H.-U., E. BERNT und B. HEss, Lactatdehydrogenase, in: H.-U. BERGMEYER (Hrsg.), Methoden der enzymatischen Analyse, S. 736, Verlag Chemie, Weinheim/Bergstraße (1962). - 4. Zewe, V. und H. J. Fromm, J. biol. Chemistry 237, 1668 (1962).

Professor Dr. P. Siegmund 1000 Berlin 33

Arnimallee 22 